\title{
25 Research Soure \\ Cell-Mimicking Nanodecoys Protect Lung Cells by Neutralization of SARS-CoV-2 Spike Proteins
}

\section{Zhenhua Li}

North Carolina State University https://orcid.org/0000-0001-9751-0864

Phuong-Uyen C. Dinh

North Carolina State University

Kristen D. Popowski

North Carolina State University

Halle Lutz

North Carolina State University

Zhenzhen Wang

North Carolina State University

Dashuai Zhu

North Carolina State University

Shiqi Hu

North Carolina State University

Jason Lobo

University of North Carolina at Chapel Hill

Ke Cheng ( $\nabla$ ke_cheng@unc.edu)

University of North Carolina at Chapel Hill

\section{Research Article}

Keywords: SARS-CoV-2, COVID-19, ACE2, Nanodecoy, Spike Protein, Lung Spheroid Cells

Posted Date: June 30th, 2020

DOI: https://doi.org/10.21203/rs.3.rs-39099/v1

License: (c) (i) This work is licensed under a Creative Commons Attribution 4.0 International License.

Read Full License 


\section{Abstract}

Coronavirus disease of 2019 (COVID-19) caused by severe acute respiratory syndrome coronavirus 2 (SARS-CoV-2) has grown into a global pandemic, with no specific antiviral treatments or vaccines are yet approved. The viral receptor, angiotensin-converting enzyme 2 (ACE2), has been demonstrated to play an integral role in the pathogenesis of SARS-CoV-2, necessary for host cell viral entry. Inspired by this, we synthesized ACE2 nanodecoys from human lung spheroid cells (LSCs) capable of binding the Spike protein as a potential neutralization agent for SARS-CoV-2. Our results show LSC-nanodecoys has a high affinity and neutralization efficiency to both spike protein and chemically synthesized SARS-CoV-2 mimics. In addition, non-invasive inhalation therapy in mice showed successful delivery of the nanodecoy to the lungs, as well as in-vivo retention of the nanodecoys over 72 hours after a single administration. Furthermore, inhalation of nanodecoy accelerated the clearance of SARS-CoV-2 mimics from the lung and did not cause toxicity.

\section{Introduction}

Severe acute respiratory syndrome coronavirus 2 (SARS-CoV-2), the pathogen at the center of the current global pandemic, is the cause of coronavirus disease-2019 (COVID-19).1 Coronaviruses are a type of common viruses, alpha (a-) coronavirus and beta ( $\beta$-) coronavirus can infect mammals and often manifest as the common cold or gastrointestinal (GI) discomfort. Rarely, more severe and lethal forms emerge. SARS-CoV-2 is capable of wreak havoc on the respiratory and immune system by inducing secretion of pro-inflammatory cytokines triggering an increase in alveolar edema, hypoxemia, dyspnea, and systemic inflammatory response syndrome (SIRS). 2 Like it's deadly predecessor, SARS-CoV-1 (cause of SARS in 2003) and MERS-CoV (cause of MERS in 2012), SARS-CoV-2 is an enveloped, positive-sense, $\beta$ - coronavirus with dangerously high human-to-human transmission rates, with reported R0 ranging from 2-6.3,4 Therefore, initial efforts to combat the virus was primarily centered on containment to stop the spread and to elucidate its pathogenesis.

However, as the virus continues to circulate, claiming over 350,000 lives worldwide. It is becoming undeniably evident that not only an efficacious vaccine but also therapeutic is necessary for ending this pandemic and preventing a potential second wave in the colder months ahead, which could coincide together with the typical influenza season. Researchers around the world are in an urgent race for effective therapy for COVID-19. However, currently, no therapy has been approved, and treatment remains mainly focused on palliative and symptomatic management.

Angiotensin-converting enzyme 2 (ACE2), which is present on many cell types and found in almost all tissues, is a carboxypeptidase that has been shown to play a pivotal role in host cell viral entry. SARSCoV-2 specifically attacks ACE2 presenting respiratory type II pneumocytes in the lungs and goblet secretory cells in the nasal mucosa as its primary sites of infection. 5 In this study, we exploit the virus's cell entry strategy as a Trojan horse to trick the virus. Over the past seven years, our lab has developed Lung Spheroid Cells (LSCs), from initial rodent studies to an on-going Phase 1 clinical trial 
(NCT04262167), as a cell therapy to treat lung fibrosis and inflammation.6-9 LSCs are a mixture of resident lung epithelial (containing both types I and II pneumocytes) and mesenchymal cells. As resident lung cells, they express ACE2. Toward those ends, we fabricated LSC membrane nanovesicles as ACE2 nanodecoys. Those nanodecoys, acting as cell mimics, are capable of binding SARS-CoV-2 Spike (S-) protein and trigger response from macrophages for viral elimination.

Moreover, the nanodecoys can be administered via inhalation directly to the lungs and can persist for at least up to 72 hours in the lung after a single treatment and accelerate the clearance of SARS-COV-2 mimic viruses. Altogether, our data provide a potential non-invasive therapeutic strategy for neutralizing SARS-CoV-2. Our approach is fundamentally different from the current two strategies: antiviral drugs and vaccines. The LSCs utilized in the fabrication of the nanodecoy are generated through a robust, reproducible, and scalable culturing method suitable for producing clinically applicable quantities of cell therapy products. Additionally, this nanodecoy technology is highly translatable as the parental cells are currently in the early clinical trial stage as a potential treatment for pulmonary fibrosis. 10

\section{Results}

\section{Fabrication of LSC-nanodecoys}

The overall rationale of our nanodecoy design is shown in Fig. 1a. First, LSCs and its parent cells, lung explant-derived cells (EDCs), are screened for ACE2 expression to determine the optimal cell types for nanodecoy fabrication. LSCs and EDCs were analyzed by immunoblotting (Fig. S1), immunostaining (Fig. 1b), and flow cytometry (Fig. 1c-d; Fig. S2-3) for ACE2 expression. LSCs were found to have higher ACE2 expression levels than its parent cell, EDCs. Also, confocal imaging showed that ACE2 was present on the membrane of AQP5+ type I pneumocytes and SFTPC+ type II pneumocytes (Fig. 1b), which are two subpopulations within LSCs, consistent with the literature.5,11 Furthermore, ACE2 was found to be coexpressed with other LSC makers such as EpCAM, CD90, and MUC5b (Fig. 1c-d and Fig. S2-3). Previous studies have indicated that $83 \%$ of ACE2-expressing cells in lung tissue were type II pneumocytes, suggesting that the lungs are the most vulnerable target organ to the SARS-CoV-2 virus.12 Thus, our results demonstrated that LSCs as primary resident lung cells might serve as an ideal cell type to prepare nanodecoys with a high level of ACE2 expression. LSC membrane nanovesicles (nanodecoys) were generated by serial extrusion of LSCs through polycarbonate membranes with pore sizes of 5,1 , and finally, $0.4 \mu \mathrm{m}$ using a commercial extruder (Fig. 1e). The obtained nanodecoys were characterized by Nanoparticle Tracking Analysis (NTA), showing a homogeneous nanoparticles population with an average size of $320 \mathrm{~nm}$ and an average concentration of $5.51 \times 1010$ particles produced from $5 \times 106$ cells (Fig. 1f). This means that in average one LSC generated 11020 nanodecoys with high producing efficiency. Flow cytometry analysis confirmed the preservation of ACE2 on the surface of the nanodecoys as well as type II pneumocyte marker SFTPC (Fig. 1g). Furthermore, transmission electron microscope (TEM) images revealed the spherical morphology of nanodecoys (Fig. 1h). 


\section{Nanodecoys can bind and neutralize S-protein in-vitro}

After having demonstrated the presence of ACE2 on nanodecoys, we then tested its binding ability of SARS-CoV-2 S-protein. We first confirmed S-protein could bind to the nanodecoy by TEM utilizing immunogold labeling (Fig. 1h). Analysis of S-protein and varying concentration of nanodecoy co-cultures determined $50 \%$ of S-protein ( $20 \mathrm{ng}$ ) were neutralized when the nanodecoys concentration was up to 109 (Fig. 2a). Nanodecoys were designed to capture intracellular and extracellular SARS-CoV-2 and tag them for immune cell elimination or inhibit their viral infection of host cells. We first examined if nanodecoys possessed the capturing ability using Rhodamine B (RhB)-labeled S-protein. S-protein was found binding to lung cells after 4 hours of incubation (Fig. 2b). DiD-labeled nanodecoys were co-localized with Sprotein in co-culture with LSCs, suggest that the nanodecoys could recognize and competitively capture the S-protein. We also found macrophages had a greater internalization efficiency of the nanodecoys than the lung cells (Fig. 2c-f), suggesting the potential clearance of nanodecoys and their neutralized SARS-COV-2 by macrophages and/or other immune cells.

\section{Nanodecoy neutralization of SARS-CoV-2 mimic virus and protection of lung cells}

Since the mechanism of internalizing individual S-protein by the nanodecoy may be different from how it may interact with an S-protein virus, such as the real SARS-CoV-2, we fabricated an S-protein virus to mimic SARS-CoV-2. The commonly used lentivirus without S-protein was artificially modified with Sprotein to create a SARS-CoV-2-like virus (Fig. 3a). Lentiviruses were first modified with Ni nitrilotriacetate (Ni-NTA) then His-tagged S-protein was conjugated onto the lentivirus through the interaction of $\mathrm{Ni}$ with His tag to give lentivirus-spike, a SARS-CoV-2 mimicking virus (SARS-CoV-2 mimics). Immunogold labeling was used to detect S-protein on the SARS-CoV-2 mimics, and TEM results showed the presence of S- protein bound to the SARS-CoV-2 mimics indicating the successful modification of S-protein on the lentivirus (Fig. 3c). We also quantified the S-protein on the virus mimic to be approximately $879 \mathrm{pg}$ of Sprotein per SARS-CoV-2 mimics. We found that $2.16 \times 105$ of nanodecoys could bind $5 \times 105$ SARSCoV-2 mimics (2.31 SARS-CoV-2 mimics per nanodecoy) (Fig. 3b). In contrast, lentivirus without S-protein had a very low binding capacity to nanodecoy. In co-culture, SARS-CoV-2 mimics were recognized by nanodecoys and internalized by macrophages after 4 hours (Fig. 3d). Both immunocytochemistry (Fig. $3 e$ ) and flow cytometry (Fig. $3 f$ and $3 g$ ) confirmed that nanodecoys could block host cell infection by SARS-CoV-2 mimics. Naïve lentivirus was not efficient to enter lung cells (14.8\% infection rate) (Fig. $3 e$, left). S-protein modification (SARS-CoV-2 mimics) promoted entry into host cells efficiently $(73.8 \%$ infection rate) (Fig. 3e, middle), and nanodecoys significantly decreased the internalization of SARS-CoV2 mimics by lung cells (from $73.8 \%$ to $28.8 \%$ ) (Fig. 3e, right). These results suggest our nanodecoys could protect the host cells from infection by S-protein containing viruses. 


\section{In-vivo biodistribution and retention of nanodecoy after inhalation delivery}

We then studied the retention and biodistribution of nanodecoys in mice after inhalation. DiD- labeled nanodecoys were administered to mice by inhalation using a nebulizer for clinical relevance, at a dose of $1 \times 1010$ nanodecoys per kg of body weight (Fig. 4a). As shown in IVIS ex-vivo images (Fig. 4b), nanodecoys could still be found in the lungs even after 72 hours of a single treatment indicating excellent retention of inhaled nanodecoys. Corresponding immunohistochemistry of lung sections showed consistent results with IVIS imaging results (Fig. 4c-d). Furthermore, we examined tissue sections from the heart, liver, lungs, kidneys, and spleen to visualize the administered nanodecoys and studied their fate by staining different cell types. In addition to the lungs, the nanodecoys were also detected in the liver, kidney, and spleen, which might be attributed to the metabolization of the particles in the clearance process of the nanodecoys by some immune cells such as macrophages as well as the natural clearance of the nanodecoy through the body. DiD-labeled nanodecoy were shown to co-localized with APQ5+ (type I) and SFTPC+ (type II) cells. We have previously demonstrated that the nanodecoy could be internalized by macrophages in-vitro, which might aid in the elimination and removal of viruses after the nanodecoys trap them. Thus, we examined if the retained nanodecoys in the lung tissue also co-localize with CD68+ macrophages. As expected, more macrophages were found in lung tissues of treated animals, and DiDnanodecoys were co- localized in macrophages (Fig. 4f).

\section{Fast clearance of SARS-CoV-2 mimicking viruses by nanodecoys}

Finally, we tested whether inhaled nanodecoys could accelerate the clearance of SARS-CoV-2 mimics in a pivotal animal model (Fig. 5a). As indicated by ex vivo imaging (Fig. $5 \mathrm{~b}$ and $5 \mathrm{c}$ ), the amounts of SARSCoV-2 mimics were significantly reduced with the inhalation of nanodecoys. Furthermore, confocal microscopy imaging revealed that inhalation of nanodecoys accelerated the clearance of SARS-CoV-2 mimics (Fig. $5 \mathrm{~d}$ and 5e). As a safety measure, we accessed systemic cytokine levels using a cytokine array (Fig. $5 \mathrm{f}$ and $5 \mathrm{~g}$ ). In addition, nanodecoys didn't induce the change of expression level of most proinflammatory compared to control group. Furthermore, H\&E from major organs, blood hematology and biochemical parameters were detected inhalation of PBS or nanodecoys and results showed nanodecoys had no toxicity effect on mice (Fig. S4-5).

\section{Discussion}

Previous studies have indicated that ACE2 is the host receptor for the novel coronavirus (SARS-CoV-2), and viral entry of SARS-CoV-2 depends on the binding of the viral S-proteins to ACE2 on host cells.13 Therefore, inhibiting the binding of S-protein to ACE2, providing a possible treatment strategy to combat COVID-19. Inspired by this, some prior works have focused on blocking SARS-CoV-2 entry by using 
recombinant ACE2 (rACE2) protein such as rACE2 alone or rACE2 fused with an Fc fragment (rACE2Fc).14,15 However, those protein- based neutralization strategies are limited by their overall short half-life after administration. Furthermore, undesired dosage and distribution of extracellular ACE2 could have unknown toxicity effects on the body.14 Furthermore, other than ACE2, other components on cell membranes also help virus docking. Therefore, our nanodecoys will likely have a broader binding capacity than pure ACE2 proteins.

Previous works have shown several anti-microbial applications by utilizing cell membrane-based nanodecoys.16-18 For example, nanodecoys from Aedes albopictus (C6/36) cell membrane-coated gelatin nanoparticles have been developed to trap Zika Virus for preventing viral infection.19 In addition, T-cell-membrane-coated nanoparticles were used as decoys for HIV neutralization owing to the presence of T-cell surface antigens for HIV binding.20 In addition to cellular-membrane-based nanodecoys, engineered liposomes were also fabricated as decoy targets to sequester bacterial toxins that were produced during active infection in-vivo.21 Our strategy represents the first report of a potential nanodecoy treatment for COVID-19 (Fig. 1a). Those nanodecoys could be generated from human lung cells in a large scale using commercially available extrusion devices. They not only express natural ACE2 but also represent a close mimic of human lung cells, which are the main targets of SARS-COV-2.

Another concern of drug development is the potential off-target effects and undesired biodistribution. Here, we present a simple and clinically relevant method of nanodecoy delivery via inhalation using a nebulizer instead of traditional intravenous (IV) injection (Fig. 4a). Inhalation of nanodecoys resulted in the direct accumulation in the lungs, which is one of the primary sites of SARS-CoV- 2 infections. Even from one single inhalation treatment, DiD- labeled nanodecoys can still be found in the lungs after 72 hours (Fig. 4b). We also detect nanodecoys in the liver, kidney, and spleen throughout the 72 hours, which can be attributed to the metabolization of nanodecoy, potentially by macrophages. The ability of the nanodecoys to be recognized and captured by macrophages would contribute to the clearance of SARSCoV-2 from circulation. Furthermore, we demonstrated that inhalation of nanodecoys could accelerate the removal of SARS-CoV-2 mimics from lungs of infected mice. We provide evidence that neutralization of SARS-CoV-2 with nanodecoy can be achieved, but further studies are necessary to test the efficacy of the nanodecoys against the genuine SARS-CoV-2, instead of S-protein or synthesized SARS-CoV-2 mimics. In addition, our current study did not use the transgenic human ACE2 mice which are more prone to the human SARS-COV-2.22 Nonetheless we provide the first evidence in vitro and in vivo that cell-derived and cell- mimicking nanodecoys can shelter lung cells from virus injection and accelerate the clearance virus in an animal model.

\section{Methods}

\section{Cell Culture}

Human macrophage primary cell (CELPROGEN) were purchased and cultured in pre-coated flasks with human macrophage primary cell culture complete extracellular matrix (Cat\# E36070-01) and media with 
serum (Cat\# M36070-01S). Human lung spheroid cell (LSC) and explant derived cell (EDC) lines were generated from healthy whole lung donors acquired from the Cystic Fibrosis and Pulmonary Diseases Research and Treatment Center at the University of North Carolina at Chapel Hill and expanded as previously described.6,7 All procedures performed in this study involving human samples were in accordance with the ethical standards of the institutional research committee and with the guidelines set by the Declaration of Helsinki.

\section{Immunoblotting and Immunostaining}

LSC and EDC cell lysate were analyzed by western blot for ACE2 (MA5-31394; Invitrogen and PA5-85139; Invitrogen) and beta-actin (MA5-15739; Invitrogen) at a 1:1000 dilution and followed by a one-hour incubation with the corresponding HRP conjugated secondary antibodies at a 1:10,000 dilution. Blots were visualized on a Bio-Rad ChemiDoc. Immunostaining was performed on cells or cryo-sectioned tissue slides fixed in $4 \%$ paraformaldehyde (PFA), which were permeabilized and blocked with Dako Protein blocking solution (DAKO; X0909) containing 0.1\% saponin (47036; Sigma-Aldrich). Cells and tissues were stained with antibodies against ACE2 (MA5-31394; Invitrogen and PA5-85139; Invitrogen), SFTPC (ab3786; Abcam), Phalloidin (ab176753; Abcam), CD4 (12-0041-82, Invitrogen), CD90 (11-0909-42, Invitrogen), and CD68 (ab955; Abcam) at a dilution of 1:100-1:200. Slides were imaged on the Olympus FLUOVIEW confocal microscope and analyzed on ImageJ (https://imagej.nih.gov/ij/).

\section{Flow Cytometry}

Cells are washed with MACS flow buffer (130-091-222; MACS) and permeabilized with BD Cytofix/Cytoperm (554714; BD) prior to incubation with antibodies against ACE2 (PA5-85139; Invitrogen), EpCAM (ab71916; Abcam), CD90 (555595; BD), MUC5b (ab77995; Abcam), and vWF (ab11713; Abcam). Nanodecoys were prepared by binding the particles to $4 \mu \mathrm{m}$ aldehyde/sulfate latex beads (A37304; Thermo Fisher) at $4^{\circ} \mathrm{C}$ overnight. The binding reaction is stopped by incubation of the nanodecoy-bead mixture with an equal volume of $200 \mathrm{nM}$ glycine for $30 \mathrm{mins}$ at room temperature followed by two washes with MACS flow buffer. Nanodecoy bond beads are then incubated with ACE2 (PA5-85139; Invitrogen) and SFPTC (AB3786; Sigma-Aldrich) antibodies for 1 hour at $4^{\circ} \mathrm{C}$ followed by two washes with MACS flow buffer. Fluorescent secondary antibodies (A32731; Thermo Fisher) are then incubated for 1 hour in the dark at $4^{\circ} \mathrm{C}$ followed by one wash with MACS flow buffer. Plain beads and unstained nanodecoy bond beads were used as controls. Flow cytometry was performed on the CytoFlex (Beckman Coulter; https://beckman.com) and analyzed using FCS Express V6 (De Novo Software; https://denovosoftware.com).

\section{Large-Scale Generation of Cell-Derived Nanodecoy}


Nanodecoys were prepared from LSCs by an extruder (AVESTIN LIPOSOFAST LF-50, AVESTIN, Inc). Cells were collected and suspended in PBS at a concentration of $5 \times 106$ cells $/ \mathrm{mL}$. A large volume of cells could be extruded immediately or stored at $-80^{\circ} \mathrm{C}$ until ready. The cells were processed through the extruded twice through $5 \mu \mathrm{m}, 1 \mu \mathrm{m}$, and $400 \mathrm{~nm}$ pore-sized polycarbonate membrane filters (Avanti Polar Lipids, Inc.), sequentially. The resulting nanodecoys were purified and concentrated using an ultrafiltration centrifuge tube (100 kDa MWCO; Millipore) and centrifuged at 4,500 g for $10 \mathrm{~min}$ and washed with PBS. The size and concentration of nanodecoys were measured using Nanoparticle Tracking Analysis system (Nanosight, Malvern). Nanodecoys were stored at $4^{\circ} \mathrm{C}$ for one week or placed in long-term storage at $-80^{\circ} \mathrm{C}$. The ACE2 receptor on the nanodecoys was detected using immunoblot, immunostaining, flow cytometry, and transmission electron microscopy (TEM) with immunogold labeling method prior to in-vitro and in-vivo testing.

\section{In-vitro Internalization of Nanodecoys}

Human macrophage primary cells and LSCs (104 cells $/ \mathrm{mL}$ ) were seeded in 4-well culture chamber slides (Thermo Fisher Scientific). Nanodecoys ( $1 \times 106$ cells $/ \mathrm{mL}$ ) were then labeled by DiD and incubated with macrophages and LSCs alone, as well as, a co-culture of macrophage and LSC (1:1) to mimic the in-vivo microenvironment. After 4 hours of incubation, free nanodecoys were removed and washed three times with PBS. Cells were fixed using 4\% PFA prior to immunocytochemistry staining with makers from macrophage (CD4; 12-0041-82, Invitrogen) and LSC (CD90; 11-0909-42, Invitrogen) and imaged on the Olympus FLUOVIEW confocal microscope. In addition, to quantify the internalization rate of nanodecoy by the different cells, cells and nanodecoys were cultured in T75 flask as previously described and collected for further flow cytometry analysis (CytoFlex; Beckman Coulter).

\section{In vitro Spike Protein Binding Experiments with Nanodecoys}

Recombinant spike proteins ( $\mathrm{MW}=76.5 \mathrm{kDa}$ ) were obtained from Sino Biologica. Spike proteins (10 $\mathrm{ng} / \mathrm{mL})$ were added to Nanodecoys with different concentration $(5 \times 109,1 \times 109,2 \times 108,4 \times 107,8 \times 106$, $1.6 \times 106$, and $3.2 \times 105$ ) and incubated for three hours. After that, the unbound spike protein was removed by ultracentrifugation $(100 \mathrm{kDa})$. Spike protein before and after binding to nanodecoys was measured using ELISA kit (Sino Biological SARS-COV-2 SPIKE ELISA KIT, Sino Biological) according to manufacturer's protocol to calculate neutralization efficiency.

\section{Synthesis of SARS-CoV-2 Mimicking Virus}

Spike protein (40591-V08H; Sino Biological) was conjugated to lentivirus (Cellomics Technology LLC) to create a SARS-CoV-2 mimic. His-tagged Spike protein was bind to Ni nitrilotriacetate (Ni-NTA) through the chemical interaction. NTA with mercapto group (N- [Na,Na-Bis(carboxymethyl)-L-lysine]-12mercaptododecanamide) were first reacted with 4- (N-Maleimidomethyl)cyclohexane-1-carboxylic acid 3- 
sulfo-N-hydroxysuccinimide ester sodium salt (Sulfo-SMCC) to give NTA-SMCC and then were added to the lentivirus. The NTA groups were conjugated to the lentivirus through the $-\mathrm{NH} 2$ group on lentivirus and $\mathrm{N}$ - hydroxysuccinimide ester on NTA-SMCC. The free NTA-SMCC was removed by centrifugation using an ultrafiltration tube (100 kDa MWCO; Millipore) to give SARS-CoV-2 mimicking virus (S-protein-lentivirus). The successful conjugated spike protein to lentivirus was confirmed using TEM. Briefly, SARS-CoV-2 mimics were incubated with anti-Spike protein antibodies overnight at $4^{\circ} \mathrm{C}$. Free antibodies were removed using an ultrafiltration tube (100 kDa MWCO; Millipore) and washed three times. Spike protein on the SARS-CoV- 2 mimics were labeled with immunogold $(10 \mathrm{~nm})$ antibodies and negative stained for TEM visualization. The gifting efficiency of spike protein to lentivirus were measured using ELISA. In brief, SARS-CoV-2 mimics (106 TU/mL) were lysed, and the lysates were homogenized and measured using ELISA kit (Sino Biological SARS-COV-2 SPIKE ELISA KIT, Sino Biological) according to manufacturer's protocol.

\section{SARS-CoV-2 Mimic Neutralization Experiment}

To study the binding efficiency, nanodecoys were first labeled using Dil. Then, $200 \mu \mathrm{L}$ of SARS-CoV-2 mimic $(5 \times 105)$ in $\mathrm{pH} 9.6$ coating buffer was added to each well of 96 -well plates and incubated at $4^{\circ} \mathrm{C}$ overnight for coating. In addition, lentiviruses without spike protein were also coated to the plates as a control. Following the incubation, the protein solution was removed, and the wells were washed with $1 \times$ PBS. To study binding, plates were incubated with Dil-labeled nanodecoy at concentrations of $1 \times 104$, $2 \times 104,4 \times 104,8 \times 104,1.6 \times 105,3.2 \times 105,6.4 \times 105,1.28 \times 106$ for two hours at room temperature.

Afterward, the plates were rinsed with $1 \times$ PBS for three times, and the fluorescent intensity was measured using a microplate reader (Molecular Devices).

Interaction of SARS-CoV-2 mimic with LSCs was assessed by ICC and flow cytometry. We studied the interaction of lentivirus-spike with LSCs. LSCs (104 cells $/ \mathrm{mL}$ ) were seeded in 4-well culture chamber slides. Lentivirus (104 TU/mL), RhB-labeled SARS-CoV-2 mimic (104 TU/mL), RhB-labeled SARS-CoV-2 mimic $(104 \mathrm{TU} / \mathrm{mL})+$ nanodecoys (105) were incubated with LSCs, respectively. After a four hours incubation, the free SARS-CoV-2 mimic were removed and washed using PBS for three times. Cells were fixed with 4\% PFA and stained for LSC (FITC-CD90; 11-0909-42, Invitrogen) were stained, and cells were imaged on the Olympus FLUOVIEW confocal microscope. Internalization rate of SARS-CoV-2 mimics by cells by flow cytometry analysis (CytoFlex; Beckman Coulter).

\section{Nanodecoy capture SARS-CoV-2 mimicking virus in cells}

We studied if nanodecoy could recognize and SARS-CoV-2 mimic in vitro, macrophage\&LSC (1:1) were co-cultured in 4-well culture chamber slides, and RhB-labeled lentivirus-spike (104 TU/mL) and DiDlabeled nanodecoy (105) were added. After four hours of incubation, the free RhB-labeled lentivirus-spike and DiD-labeled nanodecoys were removed and washed using PBS three times. Cells were fixed using $4 \%$ 
PFA. Cells were fixed with 4\% PFA and stained for LSC (FITC-CD90; 11-0909-42, Invitrogen) were stained, and cells were imaged on the Olympus FLUOVIEW confocal microscope.

\section{Animal Studies}

All studies and protocols were approved by the Institutional Animal Care and Use Committee at North Carolina State University. Male CD1 mice were obtained from Charles River Laboratory (Massachusetts, USA). DiD-labeled nanodecoy (1x 1010 particles per kg of body weight) was delivered to the CD1 mice via inhalation treatment using a nebulizer (Pari Trek S Portable Compressor Nebulizer Aerosol System; 047F45-LCS). Mice were imaged at 0, 24, 48, and 72 hours using Xenogen Live Imager (IVIS). In addition, mice were euthanized at 24, 48, and 72 hours. All major organs were collected, and ex-vivo images taken on the Xenogen Live Imager (IVIS). Additionally, lung tissue were cryo-sectioned for further immunofluorescence analysis of nanodecoy in-vivo biodistribution post-inhalation.

\section{In vivo clearance of the SARS-CoV-2 mimicking virus by nanodecoys}

AF647-labeled SARS-CoV-2 mimics (5x 106 per kg of body weight) was first delivered to the CD1 mice via inhalation treatment using a nebulizer (Pari Trek S Portable Compressor Nebulizer Aerosol System; 047F45-LCS). After 1 day, the nanodecoy (1x 1010 particles per kg of body weight) was inhaled and lung organs were collected and imaged at 1, 2, 3, 4, 5, and 6 days using IVIS. Additionally, lung tissue were cryo-sectioned for further analysis of SARS- CoV-2 mimics in-vivo biodistribution post-inhalation. The blood were collected for cytokine array analysis as well (Mouse Cytokine Array C1000, Raybiotech) according to the manufacture's instructions.

\section{In vivo safety evaluation}

CD1 mice were treated by PBS and nanodecoy (1x 1010 particles per kg of body weight) through inhalation. After 14-day treatment, the blood (blood test) and major organs (H\&E) were collected for safety evaluation.

\section{Statistical analysis}

All experiments were performed at least three times independently. Results are shown as means \pm standard deviation. Comparisons between any two groups were performed using the two- tailed, unpaired Student's t-test. Comparisons among more than two groups were performed using one-way ANOVA, followed by the post hoc Bonferroni test. Single, double, and triple asterisks represent $p<0.05,0.01$, and 0.001 , respectively; $p<0.05$ was considered statistically significant. 


\section{Declarations}

\section{Data Availability}

The data that support the findings of this study are available from the corresponding author upon reasonable request.

\section{Author Contributions}

Z.L. and P.U.C.D. contributed equally to this work. Z.L. and K. C. designed the study. Z.L. and P.U.C.D. performed the experiments, interpreted the results, and wrote the paper. K.D.P. and H.L. helped with ICC and western blot. Z. W., D.Z., and S.H. helped with flow cytometry and analyzed that data. K.C. helped conceive and design the study, supervised the experiments, helped edit the paper and provided funding support.

\section{Competing Interests}

K.C. is a co-founder and equity holder of BreStem Therapeutics. P.U.C.D. reports compensation for consulting from BreStem Therapeutics. BreStem provided no funding to this study. The remaining authors declare no competing interests.

\section{References}

1. Wang, , Horby, P. W., Hayden, F. G. \& Gao, G. F. A novel coronavirus outbreak of global health concern. Lancet (London, England) 395, 470-473 (2020).

2. Wang, et al. Clinical Characteristics of 138 Hospitalized Patients With 2019 Novel CoronavirusInfected Pneumonia in Wuhan, China. JAMA (2020). doi:10.1001/jama.2020.1585

3. Zhao, S. et al. Preliminary estimation of the basic reproduction number of novel coronavirus (2019nCoV) in China, from 2019 to 2020: A data-driven analysis in the early phase of the outbreak. J. Infect. Dis. 92, 214-217 (2020).

4. Yuan, , Li, M., Lv, G. \& Lu, Z. K. Monitoring transmissibility and mortality of COVID- 19 in Europe. Int. J. Infect. Dis. 95, 311-315 (2020).

5. Ziegler, C. G. K. et al. SARS-CoV-2 receptor ACE2 is an interferon-stimulated gene in human airway epithelial cells and is detected in specific cell subsets across tissues. Cell (2020). doi:https://doi.org/10.1016/j.cell.2020.04.035

6. Henry, et al. Adult Lung Spheroid Cells Contain Progenitor Cells and Mediate Regeneration in Rodents With Bleomycin-Induced Pulmonary Fibrosis. Stem Cells Transl. Med. 4, (2015).

7. Dinh, C. et al. Derivation of therapeutic lung spheroid cells from minimally invasive transbronchial pulmonary biopsies. Respir. Res. 18, (2017). 
8. Cores, J. et al. Safety and Efficacy of Allogeneic Lung Spheroid Cells in a Mismatched Rat Model of Pulmonary Fibrosis. Stem Cells Med. 6, 1905-1916 (2017).

9. Dinh, -U. C. et al. Inhalation of lung spheroid cell secretome and exosomes promotes lung repair in pulmonary fibrosis. Nat. Commun. 11, 1064 (2020).

10. Cores, J. et al. A pre-investigational new drug study of lung spheroid cell therapy for treating pulmonary fibrosis. Stem Cells Med. (2020). doi:10.1002/sctm.19-0167

11. Hamming, et al. Tissue distribution of ACE2 protein, the functional receptor for SARS coronavirus. A first step in understanding SARS pathogenesis. J. Pathol. 203, 631-637 (2004).

12. Zhang, H., Penninger, J. M., Li, , Zhong, N. \& Slutsky, A. S. Angiotensin-converting enzyme 2 (ACE2) as a SARS-CoV-2 receptor: molecular mechanisms and potential therapeutic target. Intensive Care Med. 46, 586-590 (2020).

13. Lan, J. et al. Structure of the SARS-CoV-2 spike receptor-binding domain bound to the ACE2 receptor. Nature 581, 215-220 (2020).

14. Lei, C. et al. Neutralization of SARS-CoV-2 spike pseudotyped virus by recombinant ACE2-Ig. Commun. 11, 2070 (2020).

15. Kruse, L. Therapeutic strategies in an outbreak scenario to treat the novel coronavirus originating in Wuhan, China. F1000Research 9, 72 (2020).

16. Fang, R.H., Luk, T., Hu, C.-M.J. \& Zhang, L. Engineered nanoparticles mimicking cell membranes for toxin neutralization. Adv. Drug Deliv. Rev. 90, 69-80 (2015).

17. Rao, , Tian, R. \& Chen, X. Cell-Membrane-Mimicking Nanodecoys against Infectious Diseases. ACS Nano 14, 2569-2574 (2020).

18. Zhang, Q., et al. Cellular Nanosponges Inhibit SARS-CoV-2 Nano Lett. (2020). doi: 10.1021/acs.nanolett.0c02278

19. Rao, , et al. A Biomimetic Nanodecoy Traps Zika Virus To Prevent Viral Infection and Fetal Microcephaly Development. Nano Lett. 19, 2215-2222 (2019).

20. Wei, , et al. T-Cell-Mimicking Nanoparticles Can Neutralize HIV Infectivity. Adv. Mater. 30, 1802233 (2018).

21. Henry, D., et al. Engineered liposomes sequester bacterial exotoxins and protect from severe invasive infections in mice. Nat. Biotechnol. 33, 81-88 (2015).

22. Sheahan, P., et al. An orally bioavailable broad-spectrum antiviral inhibits SARS- CoV-2 in human airway epithelial cell cultures and multiple coronaviruses in mice. Sci. Transl. Med. 12, eabb5883 (2020).

\section{Figures}



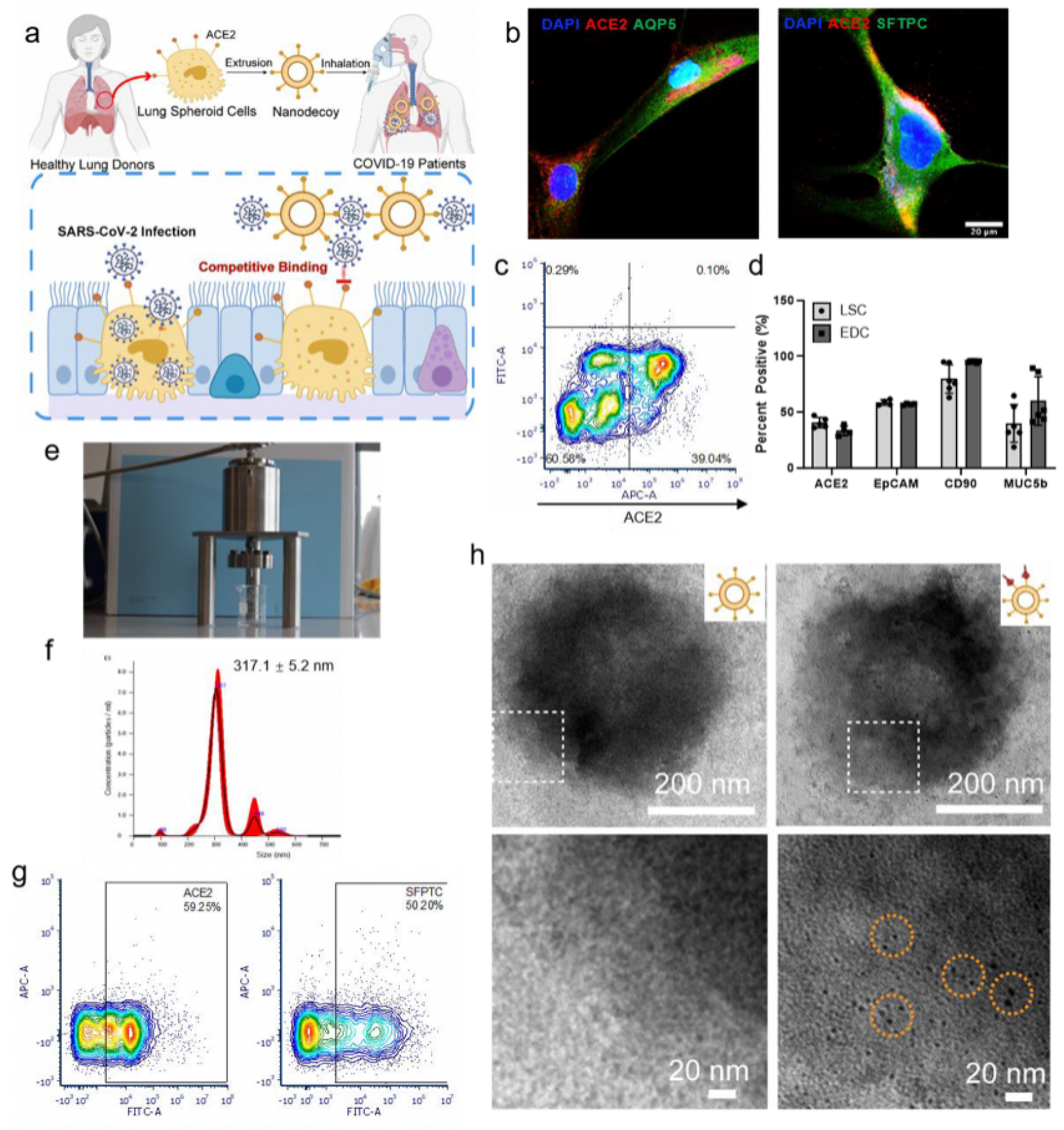

\section{Figure 1}

Generation and characterization of lung spheroid cell nanodecoy. (a) Schematic illustrating the generation of nanodecoys from lung derived cells to inhalable nanodecoys and its proposed molecular mechanism to inhibit SARS-CoV-2 entry into the host cell. (b) Representative confocal images of LSCs labeled with ACE2, AQP5, and SFTPC. (c) Representative flow cytometry analysis of LSCs for ACE2 and (d) quantitative results. (e) Photograph of the extrusion device used to generate the nanodecoys. (f) Size measurement of nanodecoys using Nanosight. (g) Western blot showing ACE2 blotting on nanodecoy 
lysate. Transmission electron microscopy (TEM) image of (h) nanodecoy and (i) spike protein-bound nanodecoy. Spike protein were detected using gold nanoparticle-labeled secondary antibodies with diameters $10 \mathrm{~nm}$.
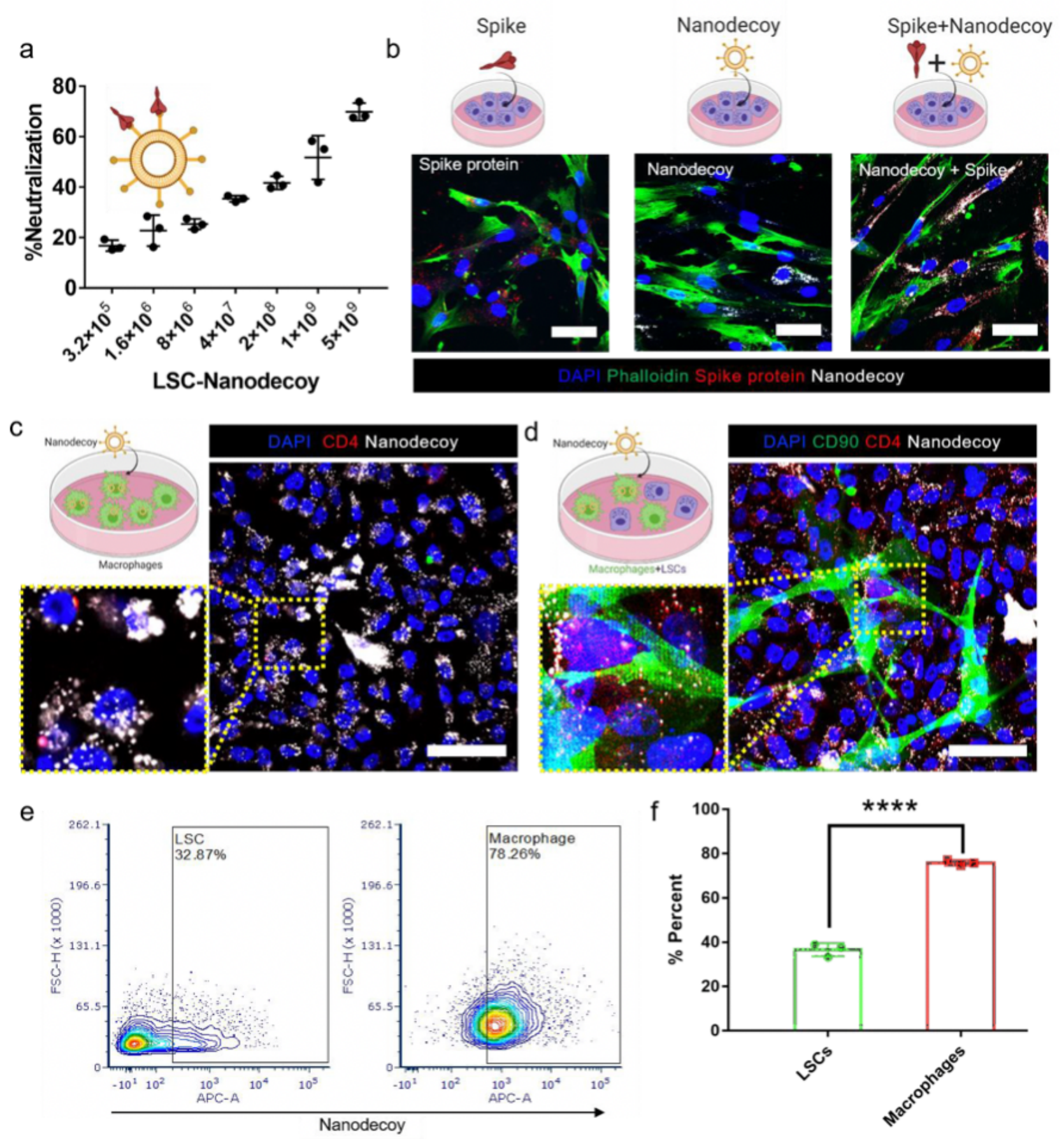

Figure 2

Neutralizing spike protein by nanodecoys. (a) Quantitative graph of neutralization efficiency of spike protein by LSC-nanodecoys. (b) Interaction of spike protein (red) and nanodecoy (white) with LSCs (green). (c) Representative confocal imaging of internalization capacity of nanodecoy by macrophages 
(red) alone or (d) a co-culture system with LSCs (green). (e) Flow cytometry analysis of internalization of DiD-labeled nanodecoys by macrophages and LSCs and (f) its corresponding quantitative graph. Scale bar, $50 \mu \mathrm{m}$.
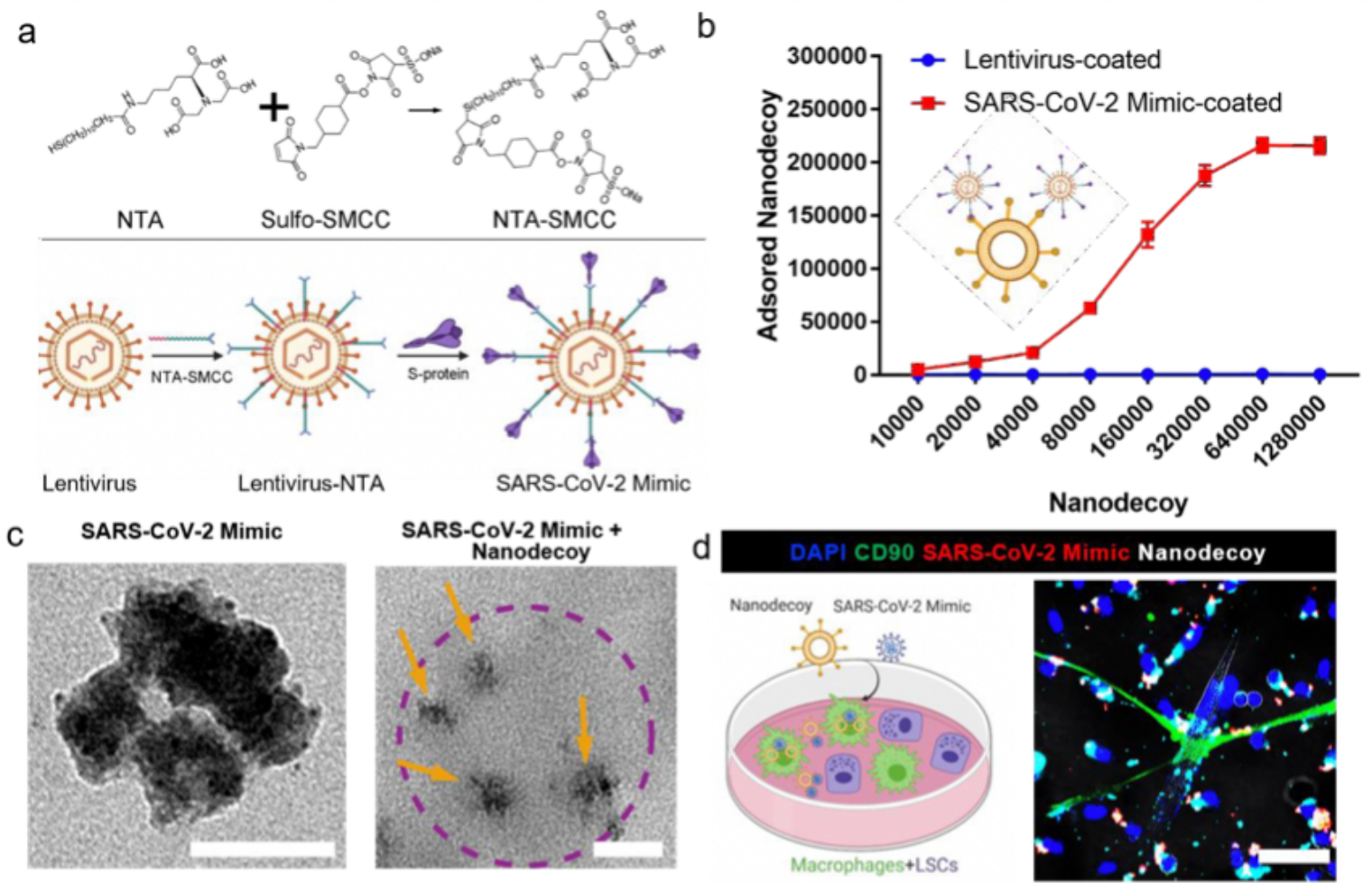

Nanodecoy
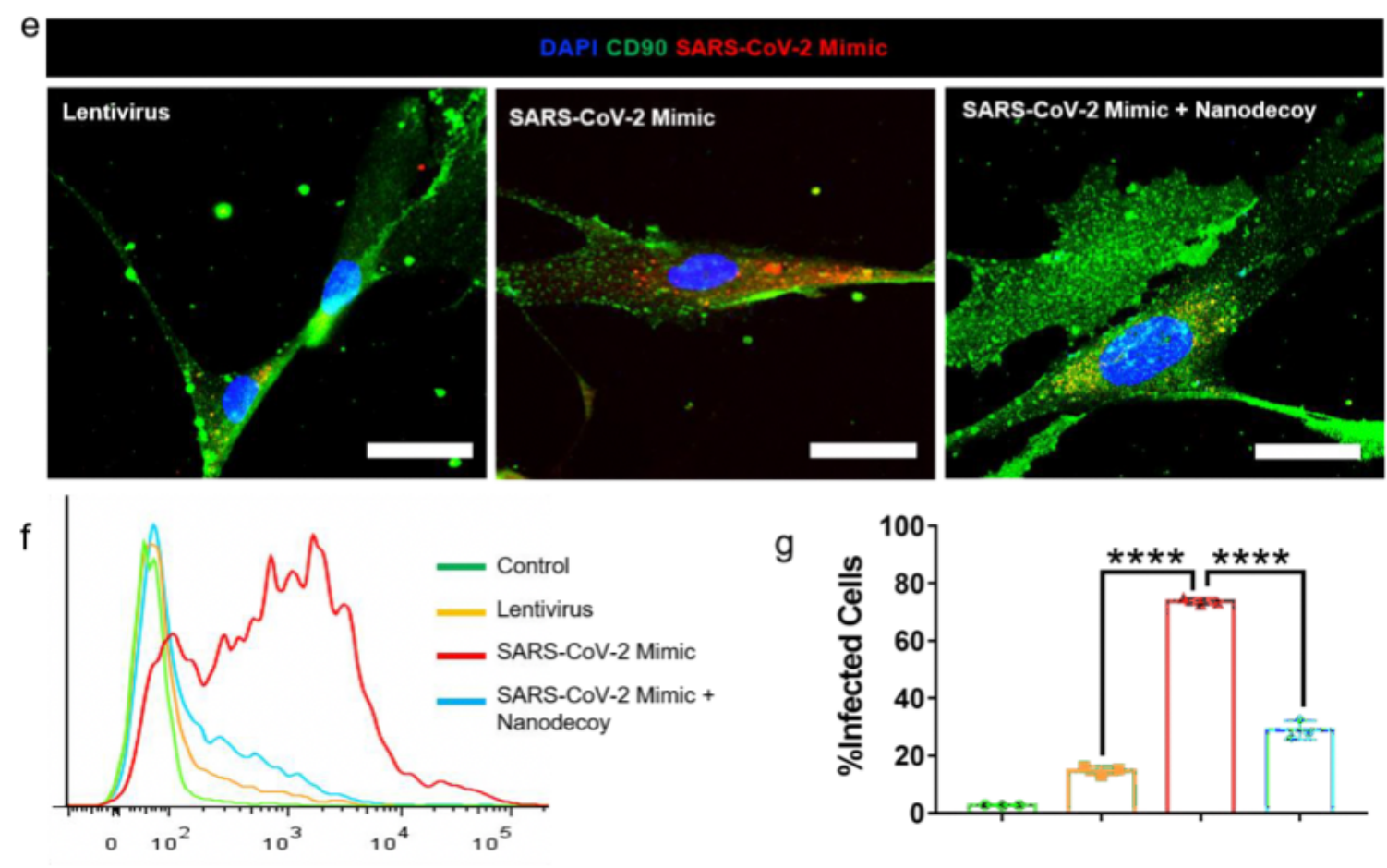

\section{Figure 3}

Nanodecoys potently neutralizes SARS-CoV-2 mimicking virus. (a) Schematic illustrating the modification of lentivirus with spike protein to generate a SARS-CoV-2 mimic. (b) Neutralization assay of SARS-CoV-2 
mimics by nanodecoys. (c) TEM image of spike protein on lentivirus using gold nanoparticle-labeled secondary antibodies with diameters of $10 \mathrm{~nm}$ and nanodecoys captured SARS-CoV-2 mimicking virus. Scale bar, $100 \mu \mathrm{m}$. (d) Nanodecoys (white) neutralizes SARS-CoV-2 mimic (red) within cells in a co-culture system. Scale bar, $50 \mu \mathrm{m}$. (e) Representative confocal imaging of nanodecoys inhibiting SARS-CoV-2 mimicking virus (red) entry into the LSCs (green). Scale bar, $50 \mu \mathrm{m}$. (f) Flow cytometry analysis of internalization of lentivirus, SARS-CoV-2 mimic, and SARS-CoV-2 mimic with nanodecoys by LSCs and (g) its quantitative results.
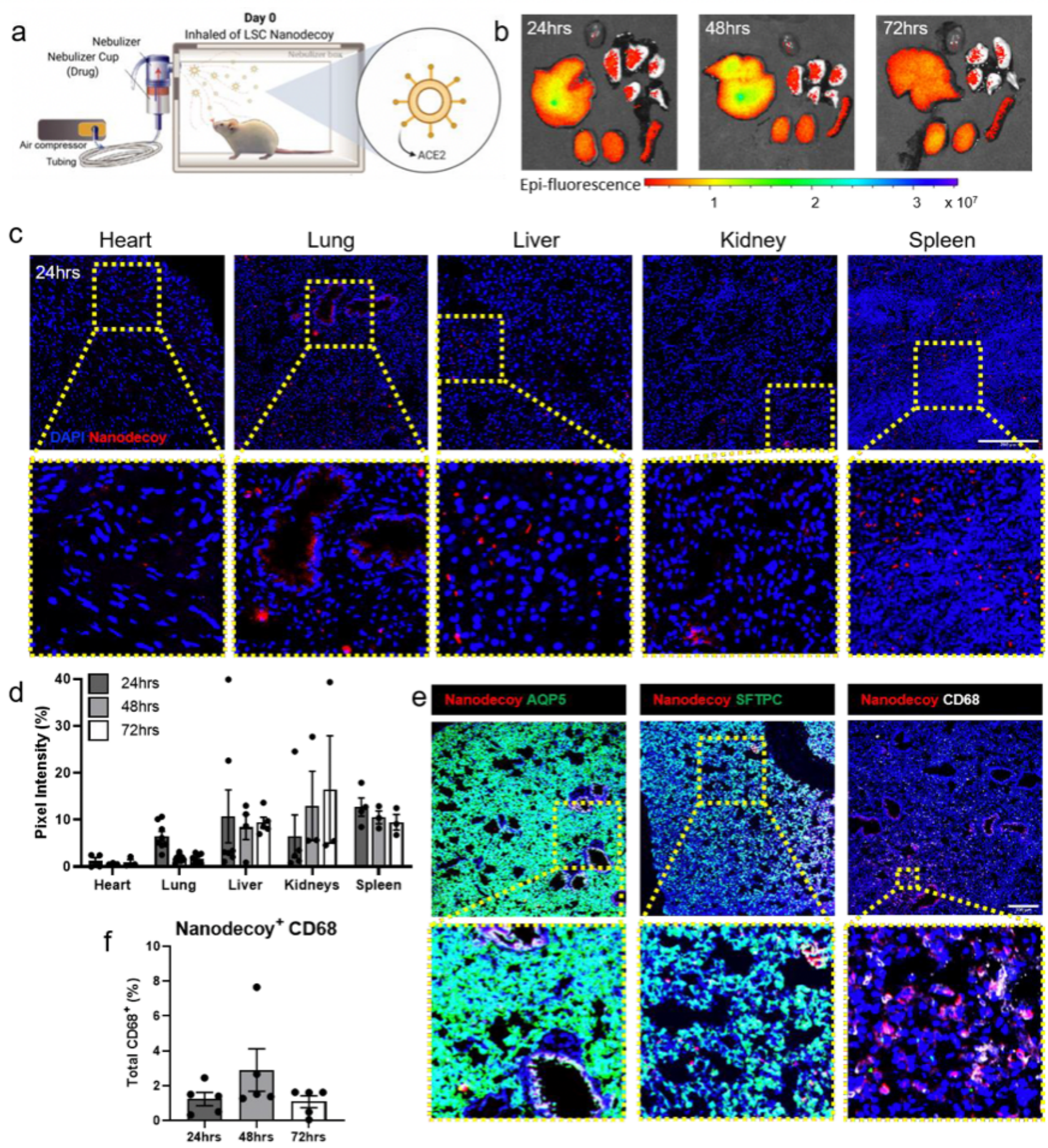


\section{Figure 4}

In vivo study of biodistribution of nanodecoy after inhalation. (a) The experimental study schematic of the nanodecoy inhalation study in CD1 mice. (b) Representative ex-vivo IVIS imaging of major tissues (from top-bottom and left-right: heart, liver, lung lobes, kidneys, and spleen) at different time points. (c) Representative confocal images of DiD-labeled nanodecoys (red) in tissue sections. (d) Corresponding quantitative graph of DiD-labeled nanodecoy in heart, lung, liver, kidneys, and spleen tissues. (e) Representative confocal imaging of nanodecoy in lung tissue section co-localizing with lung cell types and macrophages by AQP5, SFTPC, and CD68 markers, respectively. (f) Quantification of the percent of Nanodecoy positive CD68 positive cells in total CD68 positive lung tissue. 
a

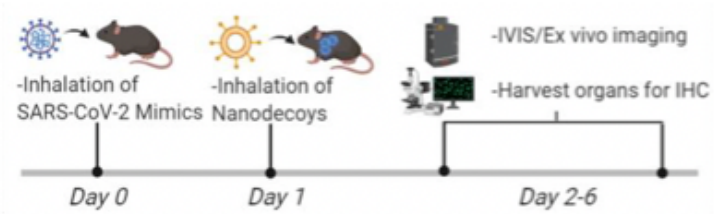

C

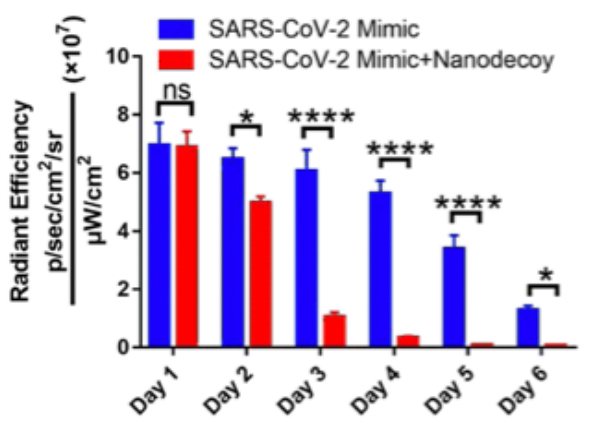

b

e
Before Day 1 Day 2 Day 3 Day 4 Day 5 Day 6
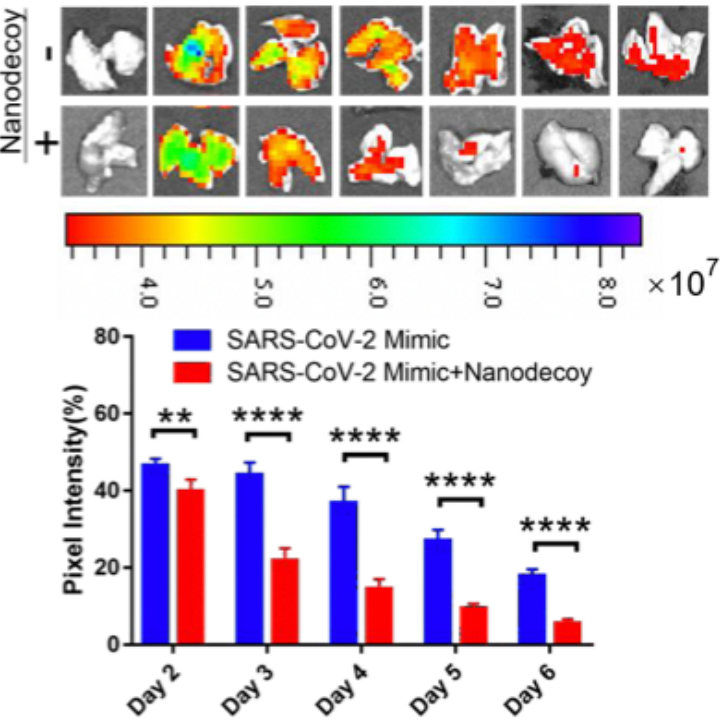

Day 4
Day 6
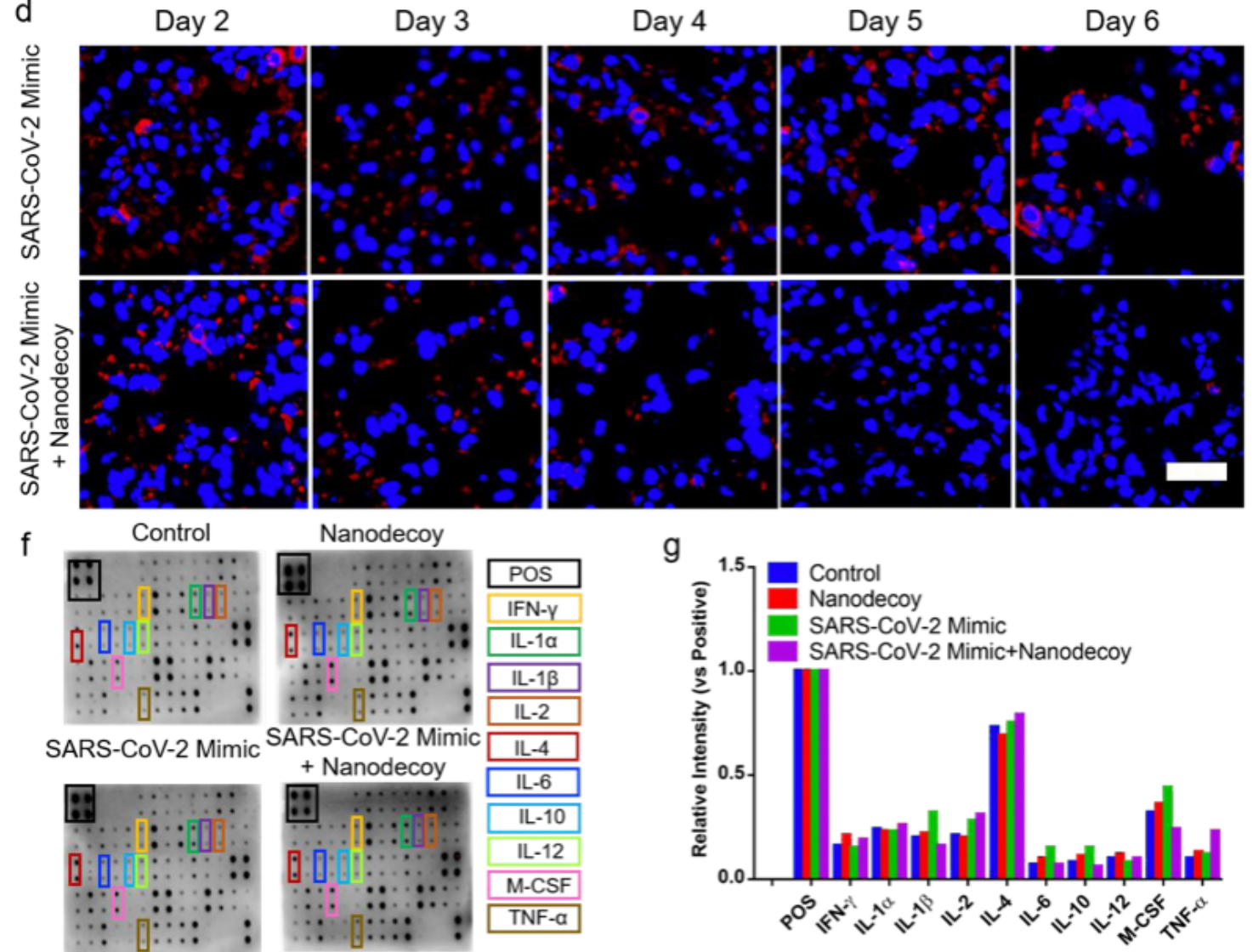

\section{Figure 5}

Nanodecoy therapy accelerates the clearance of the SARS-CoV-2 mimic viruses in mice. (a) Schematic showing animal study design. (b) Ex vivo fluorescent imaging of lungs after inhalation of SARS-CoV-2 mimics alone or SARS-CoV-2 mimics plus nanodecoy therapy (also by inhalation) at different time points.

(c) Quantification of the fluorescence intensities of SARS-CoV-2 mimics from imaging data in (b). (d) Representative confocal images of AF647-labeled SARS-CoV-2 mimics (red) in lung sections. Scale bar, 
$50 \mu \mathrm{m}$. (e) Corresponding semi-quantitative analysis of AF647-labeled SARS-CoV-2 mimics in lung tissues. (f) Cytokine array analysis of various inflammatory cytokines in the serum day 3 days after treatment. (g) Quantitative analysis of cytokine array data in (f).

\section{Supplementary Files}

This is a list of supplementary files associated with this preprint. Click to download.

- SupplementalMaterials.pdf 